\title{
Pyruvate Kinase
}

National Cancer Institute

\section{Source}

National Cancer Institute. Pyruvate Kinase. NCI Thesaurus. Code C129392.

A family of enzymes that catalyzes the transfer of a phosphate group from

phosphoenolpyruvate (PEP) to adenosine diphosphate (ADP), yielding one molecule of pyruvate and one molecule of adenosine triphosphate (ATP). This reaction is the final step of the glycolysis pathway. 